\title{
The Structure of an Allosamidin Complex with the Coccidioides immitis Chitinase Defines a Role for a Second Acid Residue in Substrate-assisted Mechanism
}

\author{
Kara Bortone, Arthur F. Monzingo, Stephen Ernst and \\ Jon D. Robertus*
}

Institute of Cellular and Molecular Biology, Department of Chemistry and Biochemistry University of Texas, Austin TX 78712, USA

\begin{abstract}
Allosamidin is a known inhibitor of class 18 chitinases. We show that allosamidin is a competitive inhibitor of the fungal chitinase CiX1 from Coccidioides immitis, with a $K_{\mathrm{i}}$ of $60 \mathrm{nM}$. We report the X-ray structure of the complex and show that upon inhibitor binding the side-chain of Asp169 rotates to form an ion pair with the oxazolinium cation. The mechanism of action is thought to involve protonation of the leaving group by Glu171 and substrate assistance by the sugar acetamido moiety to form an oxazoline-like intermediate. We converted both amino acid residues to the corresponding amide and found that each mutation effectively abolishes enzyme activity. X-ray structures show the mutant enzymes retain the basic wild-type structure and that the loss of mutant activity is due to their altered chemical properties. The high affinity of allosamidin, and its similarity to the putative reaction intermediate, suggests it is a transition state analog. This helps validate our contention that the role of Asp169 is to electrostatically stabilize the reaction transition state.
\end{abstract}

(C) 2002 Elsevier Science Ltd. All rights reserved

Keywords: chitinase; glycohydrolase; enzyme inhibition; mutation; catalytic mechanism
$2.2 \AA$ X-ray structure of a bacterial-expressed form of the CiX1, consisting of residues $36-427 . .^{5}$ Like other members of this family, CiX1 is an eightstranded $\beta / \alpha$ barrel structure with a pronounced active-site cleft at the C-terminal end of the $\beta$-barrel. The fungal enzyme structure is very similar to the family 18 chitinase from the bacteria Serratia marcescens. ${ }^{6}$ We recently characterized some kinetic properties of $\mathrm{CiX} 1$, showing it operates by a retaining mechanism, has a $\mathrm{pH}$ optimum near 6, and prefers to cleave oligosaccharides between the second and third residue from the non-reducing end.?

The exact mechanism of catalysis by family 18 chitinases is still unclear. Family 18 chitinases are thought to operate through a retaining mechanism. ${ }^{8}$ That is, the $\beta$ anomeric configuration found in the substrate is retained in the product sugar. It was originally considered that the family 18 chitinases might act like hen egg-white lysozyme, another glycohydrolase which retains the anomeric conformation of the substrate, using a general acid to protonate the leaving group and a base to stabilize the transition state. $^{6}$ Such a
Abbreviations used: GST, glutathione $S$-transferase.

E-mail address of the corresponding author: jrobertus@mail.utexas.edu 
mechanism was initially proposed for the $S$. marcescens chitinase; the corresponding active site residues conserved in CiX1 would be E171 as the acid and D240 as the base.

However, hevamine, a family 18 glycohydrolase with both chitinase and lysozyme activities, lacks the second acid residue, with an asparagine in that position. On the basis of the observed binding of the inhibitor allosamidin to hevamine, it was proposed that the family 18 chitinases uses a substrate-assisted catalytic mechanism. ${ }^{9,10}$ In this mechanism a catalytic acid, E171 in the CiX1 sequence, protonates the leaving group, and positive charge built up on the sugar at subsite -1 (the scissile glycosidic bond lies between the -1 and +1 subsites) is stabilized by interaction with its own $\mathrm{N}$-acetyl group, forming an oxazolinium intermediate. Only one catalytic group is therefore involved in this mechanism. The substrate-assistance hypothesis was given additional support by theoretical modeling studies ${ }^{11}$ and by the fact that chitin could be synthesized in vitro by a Bacillus chitinase from a chitobiose oxazoline derivative. ${ }^{12}$

Very recently, high resolution crystal structures of complexes have been observed between mutant chitinases from S. marcescens and octa- and hexasaccharide substrates. ${ }^{13}$ It was found that mutations of conserved residues Tyr390 and Asp313 (239 and 169, respectively, in CiX1) had greatly reduced enzymatic activity, and proteins with these residues mutated were used in the study. The protein-oligosaccharide complexes revealed that the sugar chain is seriously rotated and bent between sugars at subsites -1 and +1 , facilitating a distortion of the sugar at subsite -1 towards a high energy boat conformation. The authors proposed a modified substrate-assisted mechanism in which a covalent oxazoline intermediate is not formed at subsite -1 during catalysis, but instead the -1 acetamido group bends close to the ring $\mathrm{O} 4$ and presumably helps neutralize developing charge. A role was proposed for the conserved Asp residue (169 in CiX1) in which it assists the catalytic acid/base Glu (171 in CiX1) but does not interact directly with the substrate.

Here we show the binding of the trisaccharide analog inhibitor allosamidin to $\mathrm{CiX} 1$, and also examine the structure and properties of several active-site mutant proteins that are important in defining the catalytic mechanism. The results of this study implicate a direct role for a second acid residue (Asp169 in CiX1) in catalysis by the class 18 family of glycohydrolases.

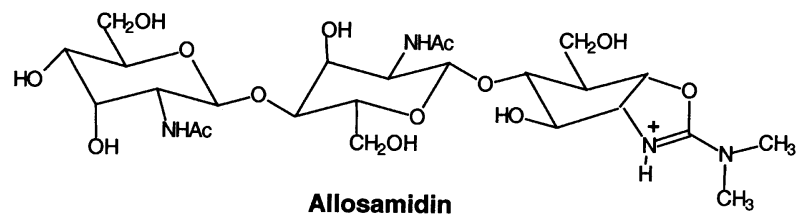

\section{Results}

\section{Inhibition by allosamidin}

The effectiveness of allosamidin as an inhibitor of CiX1 was initially approximated by measuring activity of $2.1 \mathrm{nM}$ enzyme with $50 \mu \mathrm{M}$ substrate in the presence of varying concentrations of allosamidin. This indicated that roughly $30 \mathrm{nM}$ inhibitor reduced activity about 50\%. Complete steady-state kinetic parameters were then measured for the enzyme in the presence of increasing concentrations of allosamidin, using the modified fluorescent assay, and gave the results shown in Figure 1(a). The modified assay clearly produces hyperbolic kinetics that can be readily fit to the Michaelis-Menten equation. The $K_{\mathrm{m}}$ of CiX1 for the fluorescent oligosaccharide substrate is $9.4 \mu \mathrm{M}$ and the $k_{\text {cat }}$ is $5.6 \mathrm{~s}^{-1}$. CiX1 activity is decreased with increasing allosamidin concentration. The nature of this inhibition is shown in Figure 1(b); the double reciprocal plot suggests
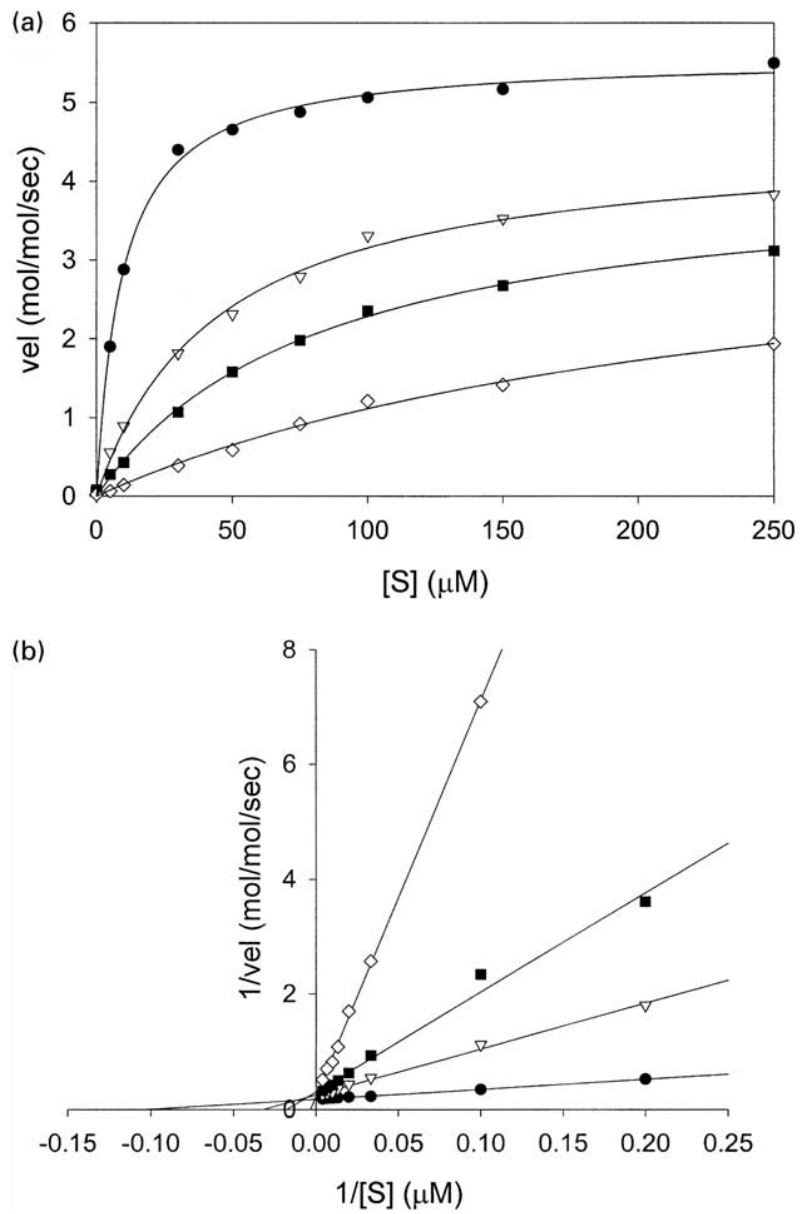

Figure 1. CiX1 activity in the presence of the inhibitor allosamidin. (a) Enzyme velocity is plotted against the concentration of fluorescent substrate in the presence of allosamidin at $0 \mu \mathrm{M}$ (filled circles), $0.1 \mu \mathrm{M}$ (open triangles), $0.5 \mu \mathrm{M}$ (filled squares), and $1.5 \mu \mathrm{M}$ (open diamonds). (b) A double reciprocal plot of the steadystate data from (a). 


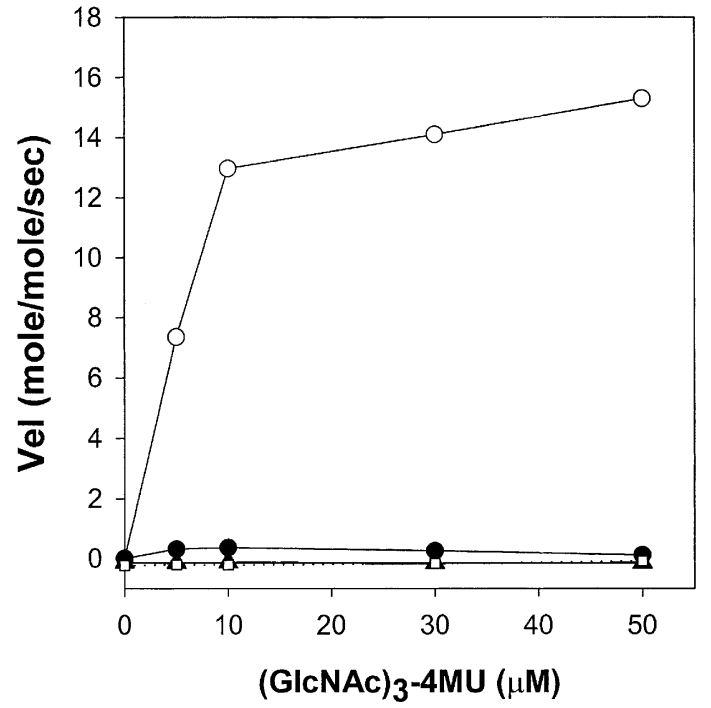

Figure 2. Kinetic activity of mutant $\mathrm{CiX} 1$ proteins. The activity of wild-type is shown as open circles, D169N by filled circles, E171Q by filled triangles, and the double mutant by smaller open squares.

that allosamidin behaves as a competitive inhibitor. A replot of the apparent $K_{\mathrm{m}}$ values (not shown) gives a $K_{\mathrm{i}}$ of $60 \mathrm{nM}$ for allosamidin.

\section{Site-direct mutants}

Primer-directed PCR was used to create several mutations at the active site of CiX1. These include converting Asp169 to Asn (D169N), Glu171 to Gln (E171Q), and the double mutant (D169N, E171Q). All of the mutant proteins were isolated by the same method used for the wild-type enzyme, and gave similar yields, roughly $30 \mathrm{mg} / 1$ of cell culture. The relative activity of the mutants was assessed using the conventional (GlcNAc) ${ }_{3}-4 \mathrm{MU}$ assay. The results are shown in Figure 2. They indicate that the $\mathrm{D} 169 \mathrm{~N}$ mutant has, at most, $1 \%$ of wild-type activity, while E171Q and the double mutant show no detectable activity.

\section{The structure of CiX1-allosamidin}

Uninhibited CiX1 crystallized in tetragonal space group $P 4_{3} 22_{1}$, with one molecule per asymmetric unit. Soaking allosamidin into these crystals caused them to crack, and so the CiX1-allosamidin complex was co-crystallized. The inhibited complex crystallizes in triclinic space group $P 1$, with four molecules in the asymmetric unit. X-ray data were collected at low temperature for the CiX1allosamidin complex to $2.8 \AA$ resolution. Crystallographic data are shown in Table 1. Molecular replacement, using native $\mathrm{CiX1}$ as a model, allowed the four molecules to be readily positioned, and omit maps of the form $2 F_{\mathrm{o}}-F_{\mathrm{c}}$ allowed the allosamidin molecules to be positioned in each of the four active sites. The packing relationship of the four $\mathrm{CiX} 1$-allosamidin complexes is shown in Figure 3. The asymmetric unit can be thought of as consisting of two dimers. Each dimer is formed from strong, symmetrical interactions across a molecular 2-fold axis; these axes are shown as arrows in Figure 3. In forming the tetrameric asymmetric unit, two dimers arrange so that their molecular 2-folds are roughly antiparallel but are offset and do not intersect. The two molecular axes are nearly perpendicular (each within $5^{\circ}$ ) to the crystallographic $a$-axis. Although the interactions within each dimer are dyadic, none of the other packing relations are symmetrical.

Because of the limited diffraction data, noncrystallographic symmetry restraints were maintained between the four monomers of the asymmetric unit during refinement, and all have essentially identical structures. The overall structure refined to reasonable crystallographic values,

Table 1. Crystallographic data

\begin{tabular}{|c|c|c|c|}
\hline & CiX1-allosamidin & D169N & E171Q \\
\hline Space group & $P 1$ & $P 1$ & $P 4_{3}$ \\
\hline Cell parameters & $\begin{array}{c}a=61.05 \AA_{b} b=78.19 \AA \\
c=88.46 \AA, \alpha=80.6^{\circ} \\
\beta=82.6^{\circ}, \gamma=66.7^{\circ}\end{array}$ & $\begin{array}{c}a=60.89 \AA_{b} b=78.18 \AA \\
c=88.43 \AA, \alpha=80.5^{\circ} \\
\beta=82.1^{\circ}, \gamma=67.0^{\circ}\end{array}$ & $\begin{array}{c}a=b=90.33 \AA \\
c=95.63 \AA\end{array}$ \\
\hline Molecules/AU & 4 & 4 & 2 \\
\hline Resolution (§) & 2.8 & 2.8 & 2.0 \\
\hline$R_{\text {merge }}(\%)$ & 13.1 & 10.6 & 5.2 \\
\hline$R_{\text {merge }}$ (last shell) (\%) & 36.9 & 22.1 & 13.3 \\
\hline$I / \sigma$ & 5.6 & 6.2 & 14.0 \\
\hline$I / \sigma$ (last shell) & 2.3 & 2.4 & 5.1 \\
\hline Completeness (\%) & 95.8 & 94.0 & 91.6 \\
\hline Unique reflections & 34,859 & 34,083 & 47,458 \\
\hline Redundancy & 2.4 & 1.7 & 2.1 \\
\hline$R_{\text {working }}$ & 0.197 & 0.182 & 0.207 \\
\hline$R_{\text {free }}$ & 0.258 & 0.256 & 0.267 \\
\hline \multicolumn{4}{|c|}{ Rms deviation from ideality } \\
\hline Bonds $(\AA)$ & 0.014 & 0.014 & 0.014 \\
\hline Angles $\left({ }^{\circ}\right)$ & 2.996 & 2.978 & 2.673 \\
\hline
\end{tabular}



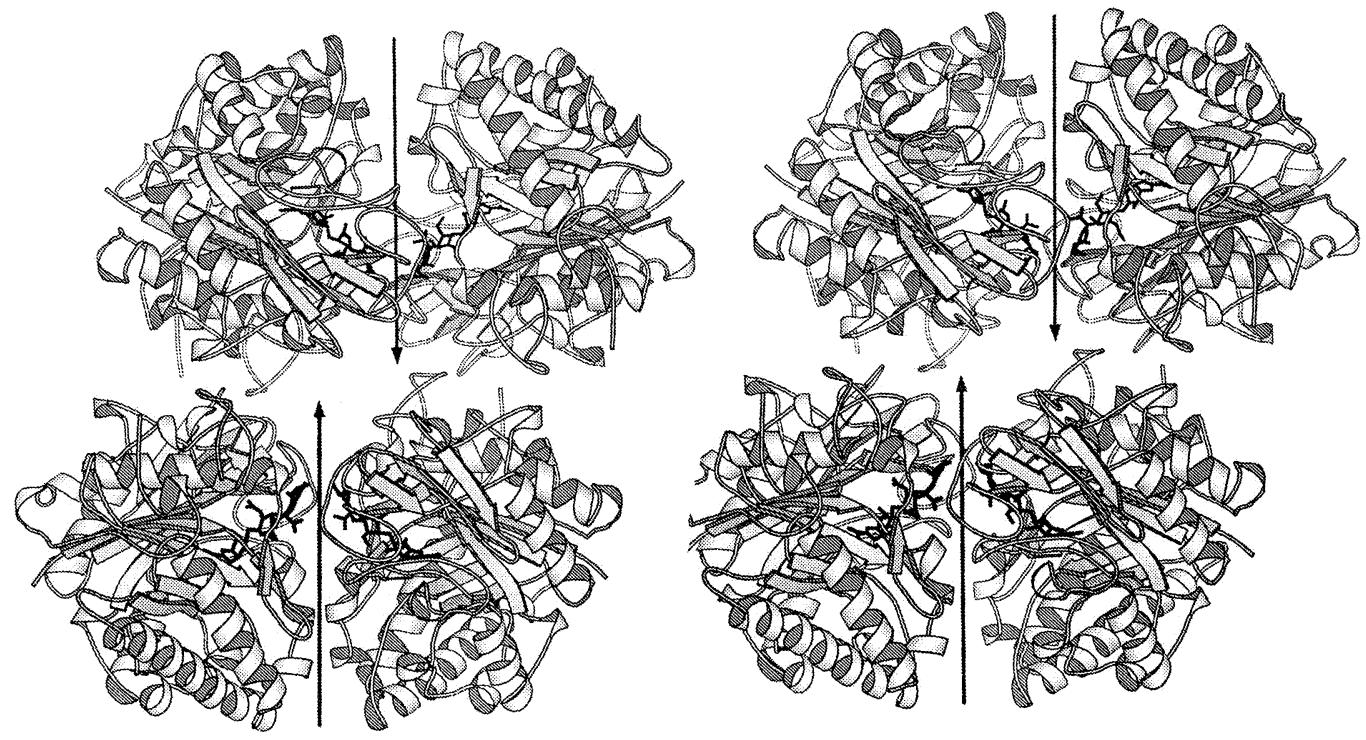

Figure 3. The crystal packing of CiX1-allosamidin. The view is down the crystallographic $a$-axis. The pseudo 2 -fold axes relating strongly interacting monomers are indicated by the arrows.

as shown in Table 1. The structure of each CiX1 monomer in the complexed crystal is very similar to the structure of apo-CiX1 described recently. ${ }^{5}$ The rms deviation of all alpha carbon atoms is less than $0.5 \AA$ between the two models. The largest differences are found in the active-site area, where CiX1 binds the inhibitor. Electron density for the inhibitor, and neighboring protein residues, in the active site of monomer 1 is shown in Figure 4. Trp residues 47 and 378 serve as a flat platform on which the sugar residues rest. The oxazoline ring of the inhibitor is bent sharply below the plane of the sugars and rests in a pocket defined by one edge of Trp378, by Tyr43 and Tyr239. Suspected catalytic residues, Asp169 and Glu171, lay along one side of this oxazoline pocket. Figure 5(a) shows some key interactions of the allosamizoline ring of allosamidin and D169 and E171. Compared with the side-chain position of the empty enzyme, the side-chain of D169 rotates roughly $100^{\circ}$ around the $C^{\alpha}-C^{\beta}$ bond so that an ion pair can be formed between the carboxylate and the positively charged oxazolinium ion of the inhibitor. The side-chain of E171 also moves slightly to form a second ion pairing with the same group. Figure $5(\mathrm{~b})$ shows a schematic of some other polar interactions between allosamidin and the enzyme; it indicates likely interactions with a carbohydrate substrate at subsites -1 to -3 .

\section{The structure of the D169N and E171Q mutants of CiX1}

As described above, the D169N and E171Q mutants of CiX1 exhibited little to no detectable enzyme activity. A common concern in mutagenesis experiments is whether the loss of activity is due to the disruption of key catalytic moieties, or if the mutation has led to improper folding of the mutant protein. Both mutant proteins crystallized in a form useful for X-ray analysis; single crystal diffraction data were collected for each, as shown in Table 1.
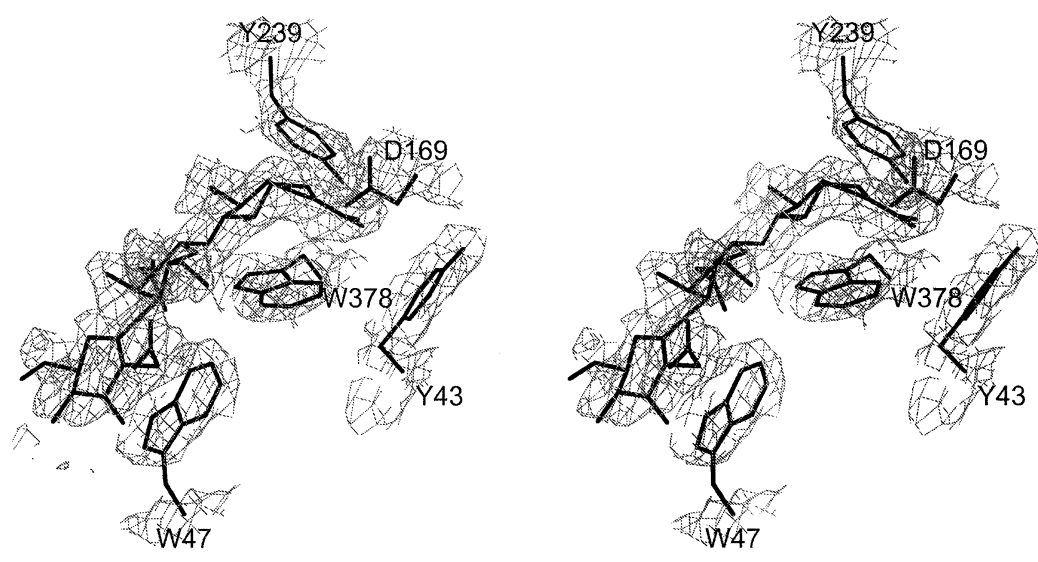

Figure 4. Superposition of allosamidin on electron density. The map is an OMIT map of the form $2 F_{\mathrm{o}}-F_{\mathrm{c}}$, contoured at $1 \sigma$ level. That is, for this map allosamidin was excluded from the phasing. Neighboring protein side-chains are displayed and labeled. 

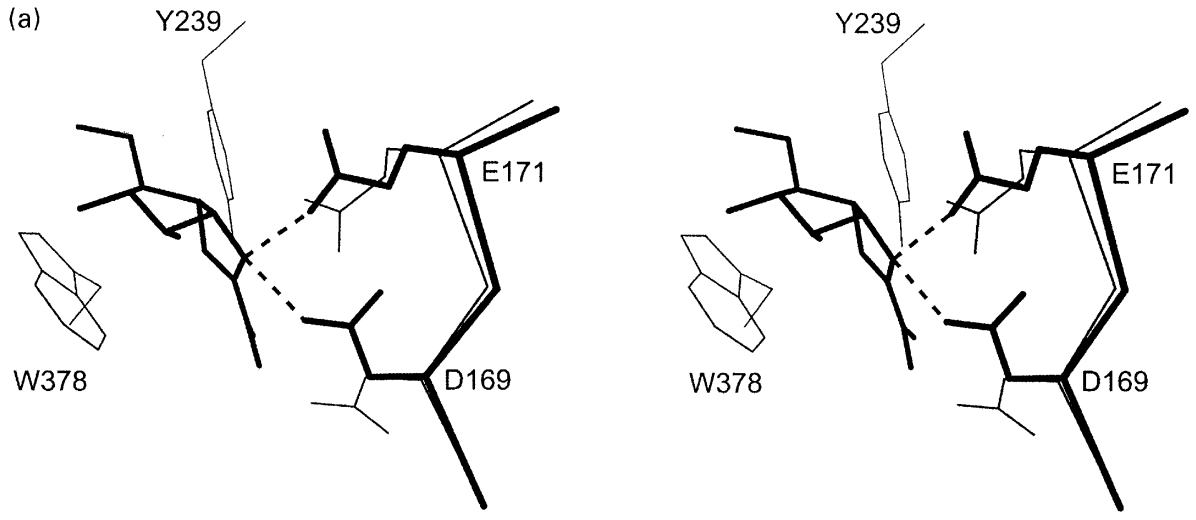

(b)

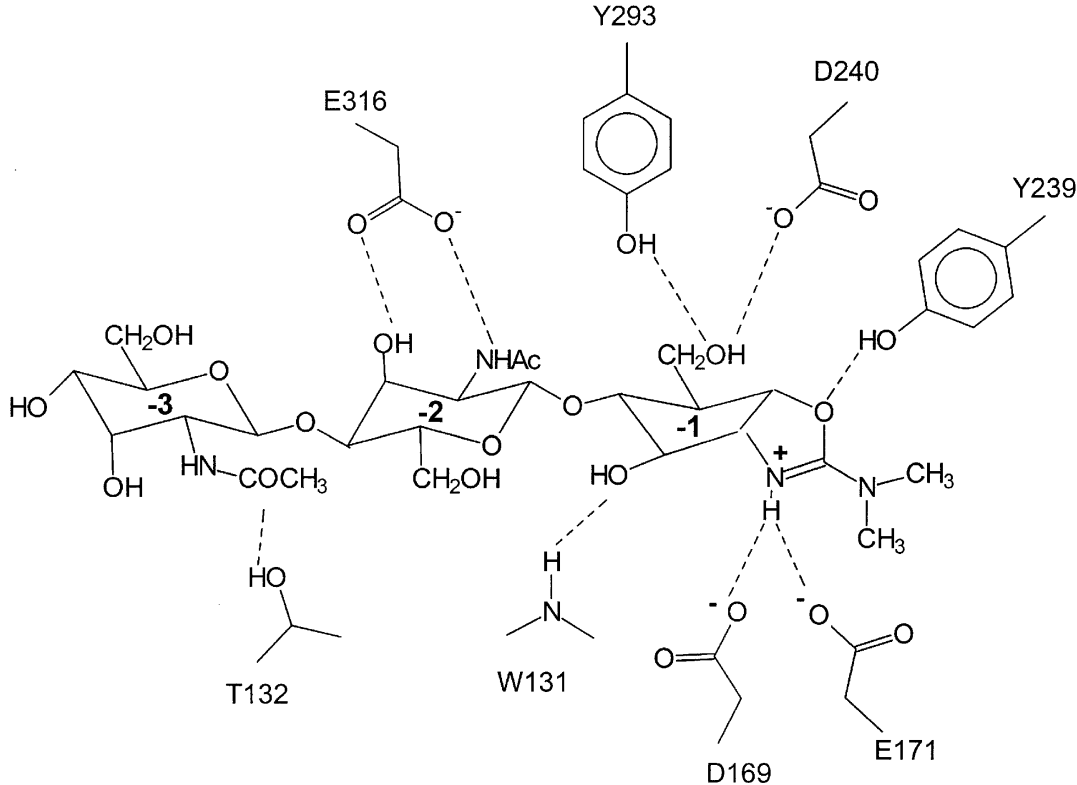

Figure 5. Interactions between allosamidin and the active site of CiX1. (a) A stereo drawing of changes in the activesite region of CiX1 upon allosamidin binding. Several key residues of the empty wild-type CiX1 enzyme are shown in thin bonds. The allosamizoline ring of allosamidin is shown in thicker bonds, as are the side-chains of D169 and E171 as observed in the complex structure. Hydrogen bonds are shown as dashed lines. (b) A schematic representation of polar interactions between allosamidin and CiX1. Allosamidin groups are marked -3 to -1 corresponding to the substrate binding site they occupy. A hydrogen bond is donated from the amido nitrogen of Trp131, but all other interactions are with amino acid side-chains, which are labeled.

Native CiX1 crystallized in space group $P 4_{3} 2_{1} 2$, with one molecule in the asymmetric unit (AU). The D169N protein crystallizes in space group $P 1$, with four molecules per AU, very much like the CiX1-allosamidin complex. The E171Q mutant crystallized in space group $P 4_{3}$, with two molecules per AU. The overall folding pattern of the various proteins is very similar, however. Because of limited resolution, the four molecules of the D169N AU were restrained to nearly identical tertiary structures. A least squares superpositioning of the D169N monomer with the parent CiX1 molecule showed the rms deviation of the corresponding $\mathrm{C}^{\alpha}$ atoms to be $0.48 \AA$; the deviation from the $\mathrm{C}^{\alpha} \mathrm{s}$ of the CiX1-allosamidin protein was $0.23 \AA$. A least squares superpositioning of the $\mathrm{A}$ and $B$ molecules of E171Q AU with native CiX1 shows rms deviations of the corresponding $C^{\alpha}$ atoms to be 0.69 and $0.56 \AA$, respectively. The $\mathrm{A}$ and $\mathrm{B}$ monomers deviate from one another by $0.63 \AA$.

The active site of the mutant CiX1 molecules is also very similar to the wild-type enzyme. Changes in the positions of the mutated residues are indicated in Figure 6. The side-chains of the 169 and 171 of the E171Q mutant are nearly superimposable with the corresponding residues of the native protein. In the D169N mutant, the mutated 169 side-chain rotates $100^{\circ}$ around the $C^{\alpha}-C^{\beta}$ bond. This is very similar to the rotation seen when allosamidin binds. There are also minor changes in the backbone near Glu171, and minor perturbations of that side-chain, so as to allow formation of a $2.9 \AA$ hydrogen bond with the amide group of the mutated, and rotated, 169 residue.

The structural analysis of the mutant proteins shows that even the complete loss of observable enzyme activity by E171Q is not due to structural 

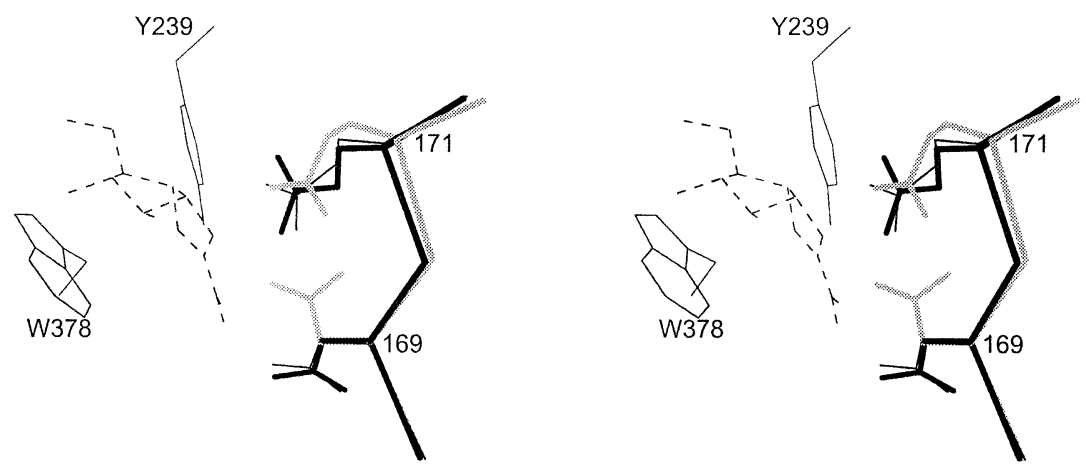

Figure 6. The active site of wildtype and mutant $\mathrm{CiX} 1$. The conformation of key active-site residues of the wild-type enzyme are shown as thin solid bonds. For reference, the position of the allosamizoline ring of allosamidin is shown in dashed lines. The conformation of the $\mathrm{C}^{\alpha}$ trace for residues $168-172$, and the side-chains of 169 and 171 for the E171Q mutant is shown as thick black bonds. The conformation of corresponding atoms of the D169N mutant is shown as thick gray bonds. changes in the mutant. The loss of activity in the $\mathrm{D} 169 \mathrm{~N}$ and $\mathrm{E} 171 \mathrm{Q}$ mutants is due to the loss of chemical function at these key positions.

\section{Discussion}

\section{Crystal forms of CiX1}

Mutant and native CiX1 have been observed to crystallize in three different crystal forms, all from very similar conditions. It is not obvious why this occurs; the mutant side-chains and bound inhibitor are not involved in any crystal packing contacts. The native protein crystallized in space group $P 4_{3} 2_{1} 2$ with one molecule per AU. The E171Q mutant crystallized with reduced symmetry in space group $\mathrm{P4}_{3}$ with two molecules per AU. A dimer, which is related by a crystallographic twofold rotation in the native crystal, is related by a rotation of $178.5^{\circ}$ in the mutant crystal. In the case of the triclinic crystal form (four molecules per AU) observed with both the allosamidin co-crystal and with the D169N mutant, dimerization occurs along a face of the protein which is not involved in dimerization in the native and E171Q crystals.

\section{Binding of allosamidin mimics the transition state}

Family 18 endochitinases bind substrate in an extended recognition site. By convention, those sugars on the non-reducing end of the substrate are said to bind in subsites given negative numbers, and those on the reducing side bind in subsites with positive numbers. In this convention, the scissile glycosidic bond lies between the -1 and +1 subsites.

Allosamidin is a strong inhibitor for family 18 chitinases; we observe a $K_{\mathrm{i}}$ of $60 \mathrm{nM}$ compared with a $K_{\mathrm{m}}$ value for similarly sized substrates, of $10 \mu \mathrm{M}$. The binding we observed for allosamidin sugars probably reflects the interactions of substrates at binding subsites -3 and -2 . As indicated in Figure 5(b), a number of hydrogen bonds help to solvate the sugars. Non-polar interactions are made between Trp378 and sugar faces at sites
-1 and -2 and between Trp47 and sugar faces in sites -2 and -3 . Because hydrogen bonds between the enzyme and the sugar hydroxyls are likely to replace those made in solution, it is likely that these non-polar contacts may be the key driving force in substrate binding.

The binding of the allosamizoline ring at site -1 probably reflects the interactions of a transition state mimic, and it is this strong interaction that accounts for the high affinity for allosamidin. We have previously made kinetic measures and model fitting using hexasaccharides, which indicated that interactions at subsite -2 are favorable by about $-3.8 \mathrm{kcal} / \mathrm{mol}^{7}$ This same fitting suggested that interactions at site -1 are unfavorable by about $3.1 \mathrm{kcal} / \mathrm{mol}$, and this is consistent with distortion to a boat conformation.

Such a distortion of the substrate toward the transition state upon binding is a common feature of enzyme mechanisms. Indeed it is generally considered that many enzymes have evolved to preferentially bind the transition state geometry, and that this accounts for the bulk of their rate enhancement.

Very recently, high resolution complexes have been observed between mutant chitinases from S. marcescens and octa- and hexasaccharide substrates. ${ }^{13}$ These structures indicate that the chitin substrate binds to enzyme sites -4 to +2 (subsites -5 and -6 involve crystal contacts thought to be of no biological significance). The conformation of the oligosaccharide substrate generally resembles that of extended $\beta$-linked sugars, except that the sugar chain is seriously rotated and bent between sugars at sites -1 and +1 , facilitating a distortion of the sugar at site -1 towards a high energy boat conformation.

\section{Identification of catalytic residues}

We have shown that the E171Q mutant of CiX1 is enzymatically inactive confirming the importance of an acid residue at that position. ${ }^{5}$ Similarly, Papanikolau et al. ${ }^{13}$ found the E315Q mutant of SmX to be inactive. It now seems clear that D240 (D391 in SmX) does not play a role in the catalytic reaction but may play a role in substrate binding 

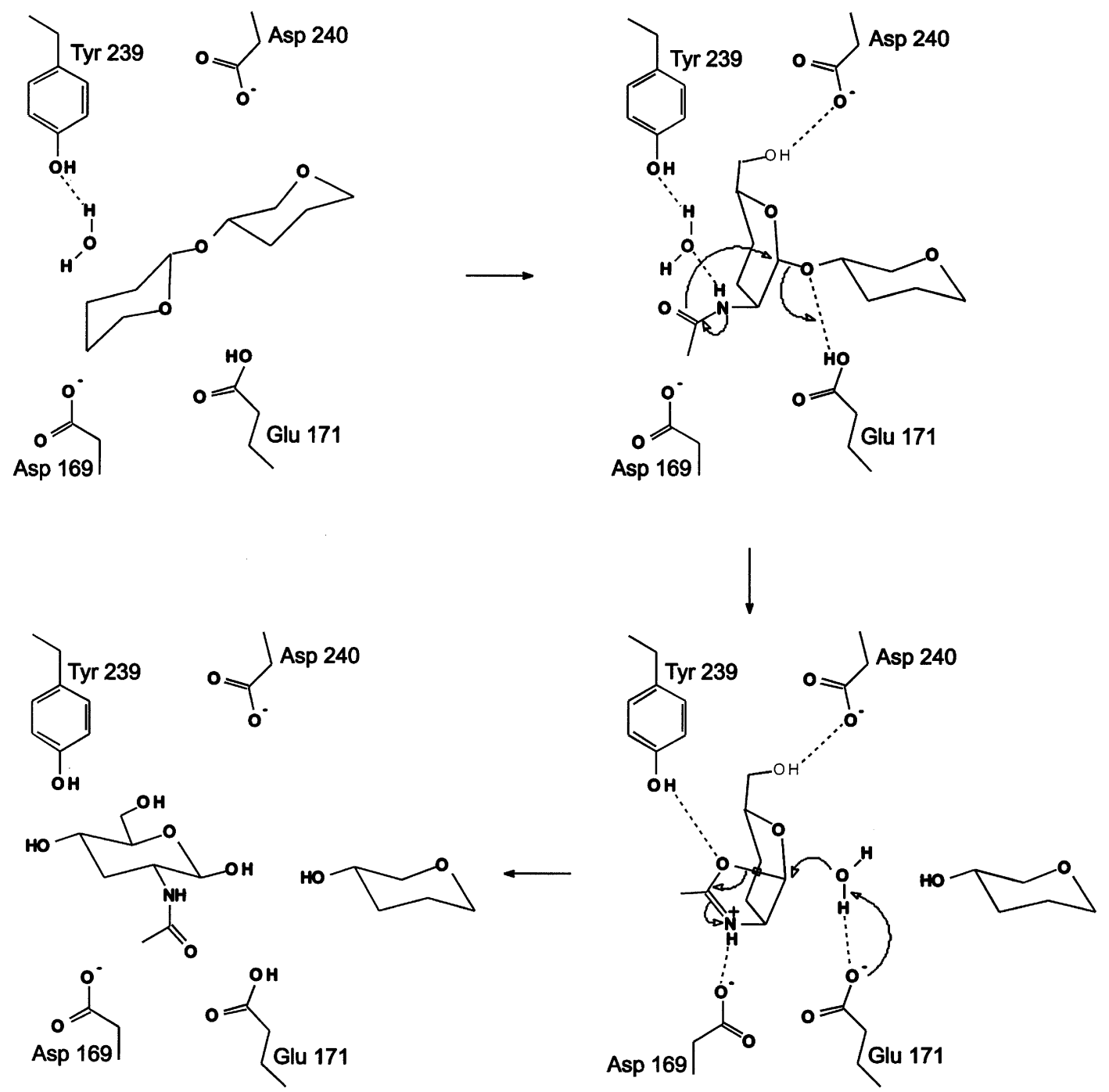

Figure 7. Proposed mechanism of catalysis. The first panel, upper left, shows the arrangement of key active-site residues and a conventional $\beta$-linked saccharide. As the substrate is bound, in the upper right panel, the polysaccharide chain is rotated and bent between sugars at sites -1 and +1 , facilitating a distortion of the sugar at site -1 towards a high energy boat conformation..$^{3}$ Two hydrogen bonds are formed with the site -1 sugar: one between a water bound to Tyr239 and the acetoamido nitrogen atom of the sugar, and the other between the sugar O6 atom and the side-chain of Asp240. A hydrogen bond is also formed between the protonated E171 side-chain and the oxygen atom of the scissile glycolytic bond. As protonation of the leaving group ( +1 site sugar) proceeds and the glycosidic bond between the sugars at -1 and +1 sites breaks, the sugar at -1 develops cationic character. This cationic character is stabilized by interaction with the -1 acetamido group, and a positively charged oxazolinium intermediate is formed. The side-chain of Asp169 rotates to form a stabilizing ion pair interaction with the cationic intermediate. A water, which may be the one displaced by the oxazolinium intermediate, then attacks the $\mathrm{C} 1$ atom of the intermediate resulting in the formation of the $\beta$-anomer.

by forming a hydrogen bond. A hydrogen bond is observed in the CiX1-allosamidin complex between a carboxylate oxygen atom of D240 and the O6 atom of the allosamizoline moiety. Papanikolau et al..$^{13}$ found the D391A mutant to be inactive with an elevated $K_{\mathrm{m}}$ but that the D391N mutant had only a slight reduction of activity. The crystal structure of the D391A mutant of SmX co-crystallized with hexa-NAG showed that the oligosaccharide was cleaved by the mutant enzyme. Furthermore, the corresponding residue in hevamine (residue 184) is asparagine, not an acid.
It has been proposed that a water molecule bound to an invariant active-site Tyr (239 in CiX1, 390 in $\mathrm{SmX}$ ) is a nucleophile during catalysis. ${ }^{13}$ The Y390F mutant of SmX has been shown to be inactive. Our structure of native $\mathrm{CiX} 1^{5}$ also shows the presence of this water, but it is displaced by the oxazoline oxygen of the bound allosamidin in the complex structure. The O7 atom of the inhibitor is found in virtually the same position in space occupied by the water in the native structure, forming a hydrogen bond with the $\mathrm{O}^{\xi}$ atom of Tyr239. It is possible that this displaced water 
attacks the catalytic intermediate to form a product that retains the $\beta$-anomer.

The role of another active-site aspartate (D169 in CiX1, D313 in SmX) has also been investigated. In the CiX1-allosamidin complex, we found a carboxylate oxygen of D169 was $2.8 \mathrm{~A}$ from the positively charged N2 atom of the allosamizoline moiety, forming an ion pair interaction. In order to determine whether an acidic residue at this position was essential for catalysis, we made the D169N mutant and found that it was catalytically inactive. The D313A mutant of SmX has also been found to be inactive. ${ }^{13}$

\section{Mechanism of catalysis}

It was originally proposed that the family 18 chitinases might act like hen egg-white lysozyme, using a general acid to protonate the leaving group and a base to stabilize the transition state. ${ }^{6}$ Those residues, in CiX1 nomenclature, would be E171 and D240, respectively. Later, it was proposed that the family 18 chitinases use a substrateassisted catalytic mechanism which requires only one catalytic group., ${ }^{9,10}$ In this mechanism, a catalytic acid, E171 in the CiX1 sequence, protonates the leaving group, and positive charge built up on the sugar at site -1 is stabilized by interaction with its own $\mathrm{N}$-acetyl group, forming an oxazolinium intermediate.

Recently, on the basis of the observed binding of oligosaccharides to mutant chitinases from S. marcescens, a modified substrate-assisted mechanism has been proposed. ${ }^{13}$ In this mechanism, a covalent oxazoline intermediate is not formed at site -1 during catalysis, but instead the -1 acetamido group bends close to the ring $\mathrm{O} 4$ and presumably helps neutralize developing charge. A role for Asp313 (D169 in CiX1) was proposed in which the Asp residue helps position the Glu residue so that it acts first as the general acid, then as the base. The aspartate does not interact directly with the substrate. The nucleophile in the hydrolytic reaction is proposed to be a water which is bound to the invariant active-site Tyr390 (239 in CiX1).

Allosamidin is a strong inhibitor for family 18 chitinases. It is likely that the allosamizoline ring of allosamidin, bound at site -1 , strongly resembles the transition state of the substrateassisted chitinase reaction mechanism, and that it interacts favorably with the enzyme for that reason. In particular, we note that ion pairs are formed between the oxazolinium cation of the ring and the side-chains of Asp169 and Glu171. The side-chain of D169 rotates roughly $100^{\circ}$ to make this interaction, but there is little additional rearrangement of the local structure.

The observation of the ion pairs leads us to suggest another variation of the chitinase catalytic mechanism, summarized in Figure 7. E171 is probably protonated prior to substrate binding. Upon binding of a natural substrate, E171 serves as a general acid to protonate the leaving group; glutamic acids serve this role in a wide range of glycohydrolases. ${ }^{14}$ As protonation proceeds, and the glycosidic bond between the sugars at -1 and +1 sites breaks, the sugar at -1 develops cationic character. This cationic character is stabilized by interaction with the -1 acetamido group and probably forms a positively charged oxazolinium intermediate. We propose that the role of Asp169 is to ion pair with that positively charged transition state in a manner similar to that observed with the allosamizoline ring of allosamidin.

This model differs significantly with the role proposed for the equivalent D169 residue in chitinase A from S. marcescens (Asp313); that model has the Asp residue acting as a part of a relay system that positions the Glu residue so that it acts first as the general acid, then as the base. The Asp is not involved in a direct interaction with the substrate. ${ }^{13}$ As our mutagenesis results show, conversion of either of Asp169 or Asp171 to the corresponding amide has very little effect on the protein structure, but abolishes catalytic activity. Both residues are important to the mechanism of action.

The ultimate nucleophile in this hydrolytic reaction is water. Papanikolau et al. ${ }^{13}$ propose that the water is one observed to bind to an invariant active site Tyr (239 in CiX1). Our structure of native CiX1 ${ }^{5}$ also shows the presence of this water, but it is displaced by the bound allosamidin in the complex structure. It is possible that this displaced water attacks the catalytic intermediate to form a product that retains the $\beta$-anomer.

\section{Materials and Methods}

\section{Protein purification}

The enzyme was purified from a fusion with glutathione $S$-transferase (GST) ${ }^{3}$ as described previously. ${ }^{15}$ Mutants of CiX1 were purified by the same method as the wild-type enzyme.

\section{Kinetic assays}

CiX1 activity was measured by its ability to hydrolyze 4-methylumbelliferyl- $\beta-\mathrm{D}-N, N^{\prime}, N^{\prime \prime}$-triacetylchitotriose $(\mathrm{GlcNAc})_{3}-4 \mathrm{MU}$ (Sigma, St. Louis, MO), releasing the fluor, 4-methylumbelliferone. Free umbelliferone is measured in fluorescence units using fluorescence spectrophotometry, exciting at $\lambda=360 \mathrm{~nm}$ and measuring emission at $\lambda=415 \mathrm{~nm}$, with a Turner ${ }^{\circledR}$ Model 450 Fluorimeter (Barnstead/Thermolyne, Dubuque, IA). Relative activities, measured as $k_{\text {cat }} / K_{\mathrm{m}}$ values were obtained as previously described. ${ }^{7} \mathrm{CiX} 1$ concentrations were generally $2.1 \mathrm{nM}$, and substrate concentrations varied from 0 to $250 \mu \mathrm{M}$, in a total volume of $350 \mu \mathrm{l}$ at $\mathrm{pH}$ 6.0. Assays were run for 30 minutes at $30^{\circ} \mathrm{C}$, and the response remained linear for at least two hours.

It was possible to determine steady-state $k_{\text {cat }}$ and $K_{\mathrm{m}}$ values using a novel, modified, assay. Galactosyl transferase catalyzes the transfer of UDP-galactose to the 
reducing end of GlcNAc: (GlcNAc) $)_{3}-4 \mathrm{MU}+\mathrm{UDP}-\mathrm{gal} \rightarrow$ gal-(GlcNAc) ${ }_{3}-4 \mathrm{MU}+\mathrm{UDP}$.

Assays were conducted using the same CiX1 and substrate concentrations as above, but including $5 \mu \mathrm{M}$ $\mathrm{MnCl}_{2}, 0.25 \mathrm{U}$ galactosyl transferase, and $0.1 \mathrm{mg}$ UDPgalactose in $0.1 \mathrm{M}$ Tris ( $\mathrm{pH}$ 7.0). Kinetic parameters were determined by least squares fitting of experimental data to appropriate equations using the program SigmaPlot (SPSS Science, Chicago, IL). It may be that the addition of galacosides to the non-reducing end of the synthetic substrates generates a longer form that binds in only a single mode to CiX1. This is unlike the shorter commercial substrates that appear to have multiple binding modes which produce complicated steady-state kinetics that do not lend themselves to a conventional interpretation. ${ }^{7}$ Allosamidin was a gift from Eli Lilly and Company (Indianapolis, IN).

\section{Site-directed mutagenesis}

Single amino acid residue changes of suspected active site residues were made using a method based on the Quik Change ${ }^{\circledR}$ Site Directed Mutagenesis Kit (Stratagene, La Jolla, CA). Mutagenesis primers were annealed to the CiX1 expression plasmid for PCR amplification using a GeneAmp ${ }^{\circledR} 2400$ (Perkin Elmer) thermocycler. To determine the presence of the desired mutations, samples were sent to the DNA Analysis Core Facility at the Institute of Cellular and Molecular Biology (UT-Austin, Austin, TX). Plasmid DNA was purified using the Qiaprep ${ }^{\circledR}$ Spin Plasmid Kit (Qiagen, Valencia, CA). The plasmid DNA $(0.5 \mu \mathrm{g})$ and $12 \mathrm{pmol}$ of sequencing primer were placed in a $12 \mu \mathrm{l}$ reaction volume. Sequencing was performed on a Perkin Elmer/ABI Prism 377 DNA Sequencer.

\section{Crystallization}

CiX1 was crystallized under slightly different conditions than previously published. ${ }^{5,15} \mathrm{CiX} 1$ at $4 \mathrm{mg} / \mathrm{ml}$ was crystallized from $0.1 \mathrm{M}$ NaHepes, 30\% PEG 4000, and $10 \%$ isopropanol. This condition also proved successful in producing crystals of wild-type chitinase cocrystallized with $100 \mu \mathrm{M}$ allosamidin and in crystallizing active-site mutants. Allosamidin was a generous gift from the Lilly Research Laboratories (Indianapolis, IN).

For X-ray diffraction, a crystal were removed from the hanging drop using a nylon cryoloop (Hampton Research, Laguna Niguel, CA) and transferred sequentially to drops containing $30 \%, 40 \%$, and $50 \%$ PEG 400 , $0.1 \mathrm{M}$ NaHepes ( $\mathrm{pH} 7.5$ ), and any desired substrates or inhibitors. After soaking for two minutes at each PEG concentration, the crystal was transferred to a similar drop containing $60 \%$ PEG 400 for 30 minutes to one hour. The crystal was then flash-cooled in liquid nitrogen and mounted for data collection.

\section{Data collection}

Cryo-cooled crystals were subjected to X-rays generated by a Rigaku RU200 rotating copper anode (Molecular Structure Corp., The Woodlands, TX) operated at $50 \mathrm{kV}$ and $100 \mathrm{~mA}$ for $30-45$ minute exposures. Diffraction was detected on a Rigaku RAXIS IV image plate (MSC). Cooled crystals were maintained at $-150{ }^{\circ} \mathrm{C}$ using a $\mathrm{N}_{2}$ cold stream apparatus (MSC).

Crystals with a suitable diffraction pattern were indexed and analyzed for the most efficient data collec- tion scheme. HKL Suite programs, ${ }^{16}$ XDISPLAY and DENZO, were used to index the crystals and to deduce the crystal orientation. This information was entered into the program STRATEGY (Raimond Ravelli, Ultrecht University), which recommends a data collection scheme (oscillation start value and range) that yields the most complete data set for a given crystal orientation. The data were reduced using HKL Suite programs. The reflection intensities were integrated using DENZO and scaled and merged using SCALEPACK. ${ }^{16}$

Data were collected from a CiX1-allosamidin co-crystal and from crystals of the D169N and E171Q mutants of CiX1.

\section{Molecular replacement}

Molecular replacement for the CiX1-allosamidin, and mutant proteins, was performed using native $\mathrm{CiX} 1$ as a search model. The first molecular replacement solution for the CiX1-allosamidin structure, crystallized in the $P 1$ space group was found using the EPMR (Evolutionary Protein Molecular Replacement) program. ${ }^{17}$ Subsequent molecular replacement solutions were calculated using X-PLOR. ${ }^{18}$

\section{Model building and refinement}

During model refinement, non-crystallographic symmetry restraints were employed. Several rounds of model refinement were performed using X-PLOR ${ }^{19}$ and REFMAC from the CCP4 program suite. ${ }^{20,21}$ The initial model for allosamidin was obtained from the Protein Data Bank (accession number 1LLO). ${ }^{9}$ MAPMAN was used to help position bound water molecules. ${ }^{22}$

Electron density maps were generated using structure factors calculated in X-PLOR. ${ }^{22}$ Both SIGMAA weighted $F_{\mathrm{o}}-F_{\mathrm{c}}($ contoured at $2 \sigma)$ and $2 F_{\mathrm{o}}-F_{\mathrm{c}}($ contoured at $1 \sigma)$ maps were calculated with FFT. ${ }^{20}$ Maps and models were viewed using the program O. ${ }^{23}$ Initially, allosamidin was modeled in the active site of one molecule in the asymmetric unit, and transformations were applied to place allosamidin in the active sites of the other three monomers of the asymmetric unit.

The geometry of the refined models was assessed by PROCHECK. ${ }^{24}$ The rms deviations from ideality of bond lengths and angles were calculated using X-PLOR.

\section{Protein Data Bank accession numbers}

Coordinates of the CiX1-allosamidin complex model and the D169N and E171Q mutant models have been deposited in the Protein Data Bank with entry codes 1LL4, 1LL6, and 1LL7, respectively.

\section{Acknowledgments}

This work was supported by grants GM 30048 from the National Institutes of Health, the Foundation for Research, and the Welch Foundation and by support from the Center for Structural Biology.

\section{References}

1. Drutz, D. J. \& Catanzaro, A. (1978). Coccidioidomycosis. Am. Rev. Respiratory Dis. 17, 559-585. 
2. Pishko, E. J., Kirkland, T. N. \& Cole, G. T. (1995). Isolation and characterization of two chitinase-encoding genes (cts1, cts2) from the fungus Coccidioides immitis. Gene, 167, 173-177.

3. Yang, C., Zhu, Y., Magee, D. M. \& Cox, R. A. (1996). Molecular cloning and characterization of the Coccidioides immitis complement fixation/chitinase antigen. Infect. Immun. 64, 1992-1997.

4. Henrissat, B. (1991). A classification of glycosyl hydrolases based on amino acid sequence similarities. Biochem. J. 280, 309-316.

5. Hollis, T., Monzingo, A. F., Bortone, K., Ernst, S., Cox, R. \& Robertus, J. D. (2000). The X-ray structure of a chitinase from the pathogenic fungus Coccidioides immitis. Protein Sci. 9, 544-551.

6. Perrakis, A., Tews, I., Dauter, Z., Oppenheimer, A. B., Chet, I., Wilson, K. S. \& Vorgias, C. E. (1994). Crystal structure of a bacterial chitinase at $2.3 \AA$ resolution. Structure, 2, 1169-1180.

7. Fukamizo, T., Sasaki, C., Schelp, E., Bortone, K. \& Robertus, J. D. (2001). Kinetic properties of chitinase-1 from the fungal pathogen Coccidioides immitis. Biochemistry, 40, 2448-2454.

8. Armand, S., Tomita, H., Heyraud, A., Gey, C., Watanabe, T. \& Henrissat, B. (1994). Stereochemical course of the hydrolysis reaction catalyzed by chitinases A1 and D from Bacillus circulans WL-12. FEBS Letters, 343, 177-180.

9. Terwisscha van Scheltinga, A. C., Armand, S., Kalk, K. H., Isogai, A., Henrissat, B. \& Dijkstra, B. W. (1995). Stereochemistry of chitin hydrolysis by a plant chitinase/lysozyme and X-ray structure of a complex with allosamidin: evidence for substrate assisted catalysis. Biochemistry, 34, 15619-15623.

10. Tews, I., Terwisscha van Scheltinga, A. C., Perrakis, A., Wilson, K. S. \& Dijkstra, B. W. (1997). Substrateassisted catalysis unifies two families of chitinolytic enzymes. J. Am. Chem. Soc. 119, 7954-7959.

11. Brameld, K. A., Shrader, W. D., Imperiali, B. \& Goddard, W. A., III (1998). Substrate assistance in the mechanism of family 18 chitinases: theoretical studies of potential intermediates and inhibitors. J. Mol. Biol. 280, 913-923.

12. Kobayashi, S., Kiyosada, T. \& Shoda, S. (1996). Synthesis of artificial chitin: irreversible catalytic behavior of glycosyl hydrolase through a transition state analogue substrate. J. Am. Chem. Soc. 118, 13113-13114.

13. Papanikolau, Y., Prag, G., Tavlas, G., Vorgias, C. E., Oppenheim, A. B. \& Petratos, K. (2001). High resol- ution structural analyses of mutant chitinase A complexes with substrates provide new insight into the mechanism of catalysis. Biochemistry, 40, 11338-11343.

14. Monzingo, A. F., Marcotte, E. M., Hart, P. J. \& Robertus, J. D. (1996). Chitinases, chitosanases, and lysozymes can be divided into procaryotic and eucaryotic families sharing a conserved core. Nature Struct. Biol. 3, 133-140.

15. Hollis, T., Monzingo, A. F., Bortone, K., Schelp, E., Cox, R. \& Robertus, J. D. (1998). Crystallization and preliminary X-ray analysis of a chitinase from the fungal pathogen Coccidioides immitis. Acta Crystallog. sect. $D, 54,1412-1413$.

16. Otwinowski, Z. \& Minor, W. (1997). Processing of $X$-ray diffraction data collected in oscillation mode. Methods Enzymol. 276, 307-326.

17. Kissinger, C. R., Gehlhaar, D. K., Smith, B. A. \& Bouzida, D. (2001). Molecular replacement by evolutionary search. Acta Crystallog. sect. D, 57, 1474-1479.

18. Brünger, A. T. (1990). Extension of molecular replacement: a new search strategy based on Patterson correlation refinement. Acta Crystallog. sect. A, 46, $46-57$.

19. Brünger, A. T. (1987). Crystallographic $R$ factor refinement by molecular dynamics. Science, 235, $458-460$.

20. Collaborative Computational Project Number 4 (1994). The CCP4 suite: programs for protein crystallography. Acta Crystallog. sect. D, 50, 760-763.

21. Murshudov, G. N., Vagin, A. A. \& Dodson, E. J. (1997). Refinement of macromolecular structures by the maximum-likelihood method. Acta Crystallog. sect. D, 53, 240-255.

22. Kleywegt, G. J. \& Jones, T. A. (1996). xdlMAPMAN and xdlDATAMAN-programs for reformatting analysis and manipulation of biomolecular electrondensity maps and reflection data sets. Acta Crystallog. sect. $D, 52,826-828$.

23. Jones, T. A., Zou, J. Y., Cowan, S. W. \& Kjeldgaard, M. (1991). Improved methods for building models in electron density maps and the location of errors in these models. Acta Crystallog. sect. A, 47, 110-119.

24. Laskowski, R. A., MacArthur, M. W., Moss, D. S. \& Thornton, J. M. (1993). PROCHECK: a program to check the stereochemical quality of protein structures. J. Appl. Crystallog. 26, 283-291. 